\title{
MicroRNA-22E Inhibits HER-3 Protein Expression to Facilitate Metastasis of Lung Adenocarcinomas
}

\author{
Hsin-Yuan Fang', Tze-Yi Lin², Shiow-Her Chiou ${ }^{3}$, Liang-Shun Wang ${ }^{4}$, Kuan-Chih Chow ${ }^{*}$ \\ ${ }^{1}$ Departments of Surgery, School of Medicine, China Medical University, Taichung, Taiwan \\ ${ }^{2}$ Departments of Pathology, China Medical University Hospital, Taichung, Taiwan \\ ${ }^{3}$ Graduate Institute of Microbiology and Public Health, Agricultural Biotechnology Center, \\ National Chung Hsing University, Taichung, Taiwan \\ ${ }^{4}$ Graduate Institution of Clinical Medicine, Medical College, Taipei Medical University, Section of Thoracic \\ Surgery, Departments of Surgery, Taipei Medical University-Shuang Ho Hospital, Taipei, Taiwan \\ ${ }^{5}$ Graduate Institute of Biomedical Sciences and Agricultural Biotechnology Center, National Chung Hsing \\ University, Taichung, Taiwan \\ Email: ${ }^{*}$ kcchow668@gmail.com, ${ }^{*}$ kcchow@dragon.nchu.edu.tw
}

Received 17 March 2015; accepted 21 April 2015; published 23 April 2015

Copyright (C) 2015 by authors and Scientific Research Publishing Inc.

This work is licensed under the Creative Commons Attribution International License (CC BY).

http://creativecommons.org/licenses/by/4.0/

c) (i) Open Access

\section{Abstract}

MicroRNA-22 (miR-22), a short non-coding RNA that post-transcriptionally regulates mRNA stability and protein synthesis, has been shown to enhance metastatic potential and to suppress HER-3, an important mRNA marker for non-small cell lung cancer (NSCLC). However, the effect of miR-22 has not been investigated in lung adenocarcinoma (LADC), the most common type of NSCLC in the Far East. In this study, we analyzed the role of miR-22 expression in LADC patients. Expression of miR-22 was detected by reverse-transcription polymerase chain reaction (RT-PCR), and confirmed by cDNA sequencing. Signals of miR-22 in LADC sections were identified using in situ hybridization (ISH). The association between miR-22 expression and survival was evaluated by the log-rank test. Induction of miR-22 expression and the effect on HER-3 levels, as well as the subsequent cell behavior were also investigated in vitro. Two types of miR-22: miR-22 and miR22H, were detected by RT-PCR. The miR-22H had extra 13 bases, 5'-TGTGTTCAGTGGT-3', at the 3'end, and this segment was named miR-22E. Using ISH, miR-22E overexpression was detected in $225(83.0 \%)$ of 271 LADC patients. A significant difference was found in cumulative survival between patients with high miR-22E levels and those with low miR-22E levels $(p<0.0001)$. In vitro, epidermal growth factor induced miR-22, but reduced HER-3 expression. Expression of miR-22 increased cell movement ability. In conclusion, expression of miR-22 is closely associated with

\footnotetext{
"Corresponding author.
}

How to cite this paper: Fang, H.-Y., Lin, T.-Y., Chiou, S.-H., Wang, L.-S. and Chow, K.-C. (2015) MicroRNA-22E Inhibits HER-3 Protein Expression to Facilitate Metastasis of Lung Adenocarcinomas. Journal of Cancer Therapy, 6, 362-374. 
tumor recurrence, metastasis and overall survival in LADC patients by suppressing HER-3 protein expression to enhance epithelial-mesenchymal transition and metastasis.

\author{
Keywords \\ HER-3, Lung Adenocarcinoma, miR-22H, miR-22, EGFR, Epithelial-Mesenchymal Transition, \\ Metastasis
}

\title{
1. Introduction
}

Recent development of chemotherapy targeting at mutated EGFR has successfully become a major strategic treatment for the lung adenocarcinoma (LADC) [1]. The patients who responded well to tyrosine kinase inhibitors (TKIs) were primarily young Asian female non-smokers [2]. However, not all patients with the similar characteristics achieved the same treatment efficacy, suggesting that other factors were involved. Using a data mining to search EGFR-related web documents of LADC, several potential genes have been identified, including DNA repair-, mitochondria transport-, and membrane receptor-associated proteins, such as hepatocyte growth factor (HGF), HGF receptor (also known as c-MET) [3]-[9] and HER-3 (also known as v-ErbB-2 avian erythroblastic leukemia viral oncogene homolog 3, ErbB-3) [10].

HER-3 is a member of HER family [11], in which only HER-1 and HER-4 have complete membrane tyrosine kinase receptor configuration. HER-2 does not possess ligand-binding site, and HER-3 is lack of cytoplasmic tyrosine kinase activity [12], and the receptor does not transduce signals [11]. Such deficiency suggests that HER-3 may act as a decoy receptor [13] to compete with HER1 and HER-4 [14] [15]. However, using statistical software to analyze data from microarray and quantitative reverse-transcription polymerase chain reaction, expression of HER-3 mRNA has been shown to be one of the high risk genes correlated with relapse-free and overall survival of patients with non-small cell lung cancer (NSCLC) [10], though the cell type, which expresses HER-3, has not been identified.

Interestingly, microRNA-22 (miR-22), which affects mRNA stability and protein synthesis [16], suppresses HER-3 protein levels [17]. Expression of miR-22 is up-regulated by AKT, a serine/threonine kinase that relays signals from epidermal growth factor (EGF), transformation growth factor alpha (TGF- $\alpha$ ), insulin-like growth factor (IGF), fibroblast growth factor (FGF), or HGF, when growth factors bind the receptors [18] [19]. In LADC patients, cigarette smoking induces HGF overexpression in alveolar type II (ATII) pneumocytes and LADC cells [3]. HGF, via c-MET, activates focal adhesion kinase (FAK), phosphatidylinositol 3-kinase (PI3K) and AKT [4].

Recently, Kasahara et al. showed that LADC patients with higher HGF levels had worse response to TKI treatments [20]. Yano et al. demonstrated that activation of PI3K/AKT circuit could outweigh the effectiveness of gefitinib [21], suggesting that miR-22 and HER-3 might be involved in TKI resistance. In this report, we studied correlations of miR-22 and HER-3 as well as the pathophysiological regulation of their expressions in LADC cells.

\section{Materials and Methods}

Tissue specimens and cell lines. From January 2006 to December 2008, tissue specimens were collected from 271 patients with newly diagnosed lung adenocarcinoma (LADC). The stage of the disease was classified according to the new international staging system for lung cancer [22]. The Medical Ethics Committee of China Medical University Hospital approved the protocol (DMR101-IRB1-213), and written informed consent was obtained from every patient before surgery. All patients had undergone surgical resection and radical N2 lymph node dissection. After treatment, patients were routinely followed every 3 to 6 months. The average age of the male patients $(\mathrm{n}=184)$ was $61.6 \pm 15.6$ years old and that of the female patients $(\mathrm{n}=87)$ was $52.5 \pm 12.3$ years old $(p=0.0154)$. ISH was performed using a single-blind procedure. Eight NSCLC [H23, H125, H226, H838, H1437, H2009, H2087, A549 (H125 and H226 are lung squamous cell carcinoma; the others are lung adenocarcinoma)], and five breast cancer cell lines (MCF-1, MCF-7, BT-20, T47D and 60055) were used for the in vitro evaluation. The cell lines were obtained from American Type Cell Culture (http://www.atcc.org/). Cells were 
grown at $37^{\circ} \mathrm{C}$ in a monolayer in RPMI 1640 supplemented with $10 \%$ fetal calf serum (FCS), 100 I.U./ml penicillin and $100 \mu \mathrm{g} / \mathrm{ml}$ streptomycin.

Reverse transcription-polymerase chain reaction (RT-PCR). The total RNA was extracted from biopsies and cell lines by a phenol/chloroform method [3]-[9]. Following RNA extraction and synthesis of cDNA, an aliquot of cDNA was subjected to PCR to determine the integrity of -actin mRNA (3). Primers were selected using Primer3 (http://www.bioinformatics.nl/). For HER-3, the primers are: HER-3s: 5'-ATAGAAACCTGGCT GCCCGA-3' [NM_001982] and HER-3a: 5'-AGAGAGGCACTGAGGTCTGA-3'. The cDNA fragment for HER-3 was 1210 base-pair (bp). For miR-22, the primers are: miR-22 reverse: 5'-CGAATTCTAGAGCTCGAG GCAG-3' and miR-22 forward: 5'-AAGCTGCCAGTTGAAGAACTGT-3' [nts 53-74, NR_029494].

Preparation and characterization of mouse antibodies. Recombinant protein containing C-terminal amino acids 852 to 1222 of HER-3 was used to immunize BALB/c mice. Two protein bands, a 180-kDa and a 185-kDa, were detected by immunoblotting. The respective band was excised from the gel and subjected to an analysis of a MALDI-TOF.

Immunoprecipitation and immunoblotting analysis. For immunoprecipitation, protein $\mathrm{G}$ sepharose ${ }^{\mathrm{TM}}$ (Amersham Biosciences AB, Uppsala, Sweden) was pre-washed before mixing with cell lysate. The mixture was incubated at $4^{\circ} \mathrm{C}$ for $60 \mathrm{~min}$, and centrifuged at $800 \times \mathrm{g}$ for $1 \mathrm{~min}$. The supernatant was reacted with purified antibodies and protein $\mathrm{G}$ at $4^{\circ} \mathrm{C}$ for 18 hours. The reaction mixture was centrifuged at $800 \times \mathrm{g}$ for $1 \mathrm{~min}$. The precipitate was washed, and dissolved in loading buffer (50 mM Tris, pH 6.8, $150 \mathrm{mM} \mathrm{NaCl}, 1 \mathrm{mM}$ disodium EDTA, $1 \mathrm{mM}$ PMSF, $10 \%$ glycerol, $5 \% \beta$-mercaptoethanol, $0.01 \%$ bromophenol blue and $1 \%$ SDS) before eletrophoresis in polyacrylamide gels. Immunoblotting was performed following the routine protocol [3]. Briefly, after electrophoresis proteins were transferred to a nitrocellulose membrane. The proteins were probed with antibodies, and visualized by exposing the membrane to an X-Omat film (Eastman Kodak, Rochester, NY).

Slide evaluation of miR-22E expression by in situ hybridization (ISH). For ISH, three different probes, $\alpha \mathrm{miR}-$ $22 \mathrm{H}, \alpha \mathrm{miR}-22 \mathrm{~L}$ and $\alpha \mathrm{miR}-22 \mathrm{E}$, were prepared.

amiR-22H: 5'-FITC-ACCACTGAACACAACAGTTCTTCAACTGGCAGCTT-3';

amiR-22L: 5'-FITC-ACAGTTCTTCAACTGGCAGCTT-3';

amiR-22E: 5'-FITC-ACCACTGAACACAACAG-3'.

In pathological section, non-tumor lung tissue (NTLT) served as internal negative control. Slides were evaluated by pathologist without clinicopathological knowledge. A specimen was considered positive when more than $25 \%$ of cancer cells were stained (miR-22+); and negative, if less than $25 \%$ of cells were stained (miR-22-) [3] [5] [6].

Statistical analysis. Correlation of miR-22E signal with clinicopathological factors was analyzed by Fisher's exact test or Chi-Square test for trend. Survival curves were plotted using the Kaplan-Meier estimator [23]. Statistical difference in survival was compared by the log rank test [24]. Statistical analysis was performed using GraphPad Prism 5 statistics software (San Diego, CA).

\section{Results}

Expression of miR-22 was detected by RT-PCR and in situ hybridization (ISH). Expression of miR-22 was detected by RT-PCR in all four LADC cell lines (Figure 1(a)), and all five LADC samples examined (Figure 1(b)). Interestingly, two RT-PCR-amplified miR-22 DNA fragments were identified in H838 cells (Figure 1(c)). The higher molecular weight one was named miR-22H, and the lower molecular weight one was named miR-22L. The respective DNA fragments were subjected to DNA sequence analysis (by Mission Biotech, Taipei, Taiwan; www.missionbio.com.tw). Nucleotide sequence homology of the cDNA fragments was matched to the public databases using a web program (http://blast.ncbi.nlm.nih.gov/Blast.cgi).

The miR-22L (Figure 1(d)) fragment matched that of miR-22: NR_029494, Homo sapiens microRNA-22. No mutation was detected. The miR-22H fragment, however, had extra 13 bases, 5'-TGTGTTCAGTGGT-3' at the 3'-end, which were not encoded by the miR-22 gene, and this segment was named miR-22E. Based on these results, we constructed three different probes for in situ hybridization (ISH), the first one was an antisense to $\mathrm{miR}-22 \mathrm{H}$, the second one was an antisense to miR-22L, and the third one was targeting at miR-22E. A probe with scrambled antisense sequences of miR-22H was used as a negative control for ISH. As determined by ISH, miR-22H, miR-22L and miR-22E were respectively detected in 245 (90.4\%), 241 (88.9\%), and 225 (83.0\%) of 271 patients (Figure 1(e)). Because no major difference in clinicopathological parameters had been found be tween miR-22H- and miR-22L-positive patient groups, we focused on miR-22E. Expression of miR-22E was detected in 168 (62.0\%) of metastatic lymph nodes. 
(a)

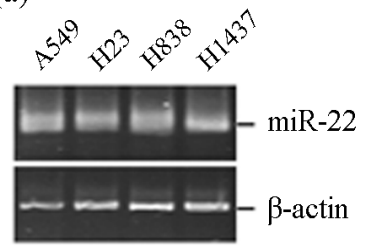

(b)

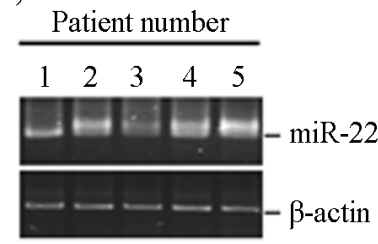

(c)
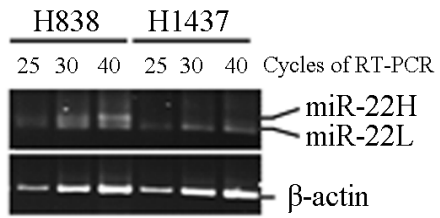

(d)

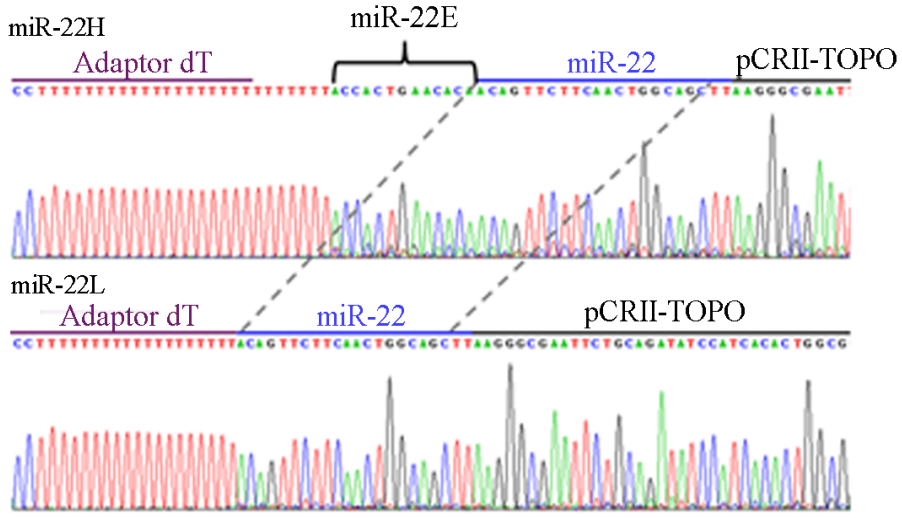

(e)

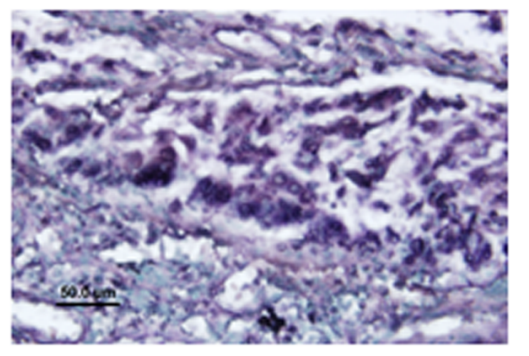

(f)

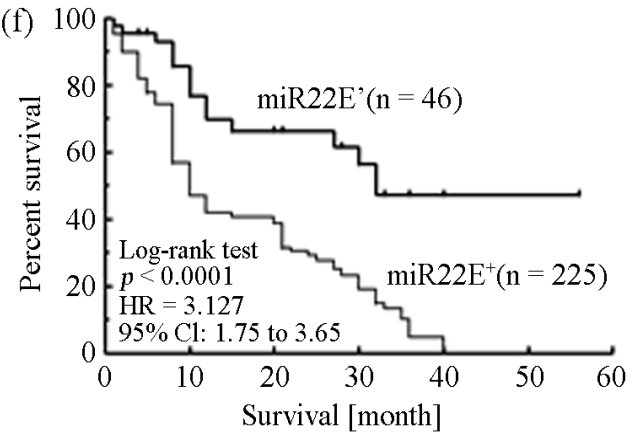

Figure 1. Detection of miR-22 expression in lung adenocarcinoma cell lines and LADC biopsies by RT-PCR and in situ hybridization. (a) Expression of miR-22 in 4 LADC cell lines as determined by RT-PCR. (b) Expression of miR-22 in 5 LADC specimens. The RT-PCR-amplified miR-22 fragments in both sample groups have two different molecular weights. (c) The two RT-PCR-amplified miR-22 products from H838 cells. The higher molecular weight one was named miR-22H, and the lower molecular weight one was miR-22L. (d) The sequence analyses of miR-22H and miR-22L (sequence matched that of miR-22: NR_029494, Homo sapiens microRNA-22). The miR-22H has extra 13 bases, 5'-TGTGTTCAGTGGT-3', at the 3'-end, which are not found within the miR-22 gene. This fragment is named miR-22E. (e) Results of in situ hybridization (ISH). The antisense oligonucleotide probes were designed to target miR-22E, and the miR-22E was mainly located in LADC cells. (f) The outcome estimates of LADC patients (Kaplan-Meier plot of overall survival). Subgroups were compared using the log-rank test. Patients who highly expressed miR-22E had significantly poorer survival rate than those who rarely expressed miR-22E (median 31 months vs. 10 months, hazard ratio [HR] = $3.127,95 \%$ confidence interval [CI], 1.75 to $3.65 ; p<0.0001)$.

Among the 225 patients who expressed miR-22E, 139 (61.8\%) patients had tumor recurrences. Among the 46 patients who had low miR-22E levels, only 12 (21.7\%) patients had metastatic lesions. All 151 patients with recurrence developed tumor within 24 months after operation. Patients with miR-22E expression were at higher risk (about 3.7-fold) of tumor recurrence. The survival rate of patients who expressed miR-22E was significantly worse than that of patients had low miR-22E expression (Figure 1(f)). The differences in cumulative survivals were significant $(p<0.0001)$. Statistical analysis showed that expression of miR-22E correlated with patient's gender, smoking habit, cell differentiation, tumor stage and lymphovascular invasion (Table 1), suggesting that miR-22E expression could be associated with cell growth and metastatic potential. 
Table 1. Comparison of clinicopathological parameters between patients with and without miR-22 expression.

\begin{tabular}{|c|c|c|c|}
\hline \multirow{2}{*}{ Parameter } & \multicolumn{2}{|c|}{ miR-22 expression } & \multirow{2}{*}{$p$ value } \\
\hline & High $(n=225)$ & Low $(n=46)$ & \\
\hline Age (yr) & $57.6 \pm 12.8$ & $56.7 \pm 11.2$ & $0.17^{\dagger}$ \\
\hline \multicolumn{4}{|l|}{ Gender } \\
\hline Male $(n=184)$ & 147 & 37 & $0.046^{\ddagger}$ \\
\hline Female $(\mathrm{n}=87)$ & 78 & 9 & \\
\hline \multicolumn{4}{|l|}{ Smoking } \\
\hline Smokers $(\mathrm{n}=195)$ & 189 & 6 & $<0.001^{\ddagger}$ \\
\hline Non-smokers $(\mathrm{n}=76)$ & 36 & 40 & \\
\hline \multicolumn{4}{|l|}{ Stage } \\
\hline I $(n=47)$ & 36 & 11 & $0.023^{\ddagger}$ \\
\hline IIa $(\mathrm{n}=66)$ & 50 & 16 & \\
\hline $\operatorname{IIb}(\mathrm{n}=132)$ & 112 & 20 & \\
\hline IIIa (n = 27) & 27 & 0 & \\
\hline \multicolumn{4}{|l|}{ Cell differentiation } \\
\hline Well $(n=26)$ & 8 & 18 & $<0.01^{\ddagger}$ \\
\hline Moderate $(n=173)$ & 146 & 27 & \\
\hline Poor $(n=72)$ & 71 & 1 & \\
\hline Mitotic index (\#/10 HPF) & $3.9 \pm 2.5$ & $5.6 \pm 3.2$ & $<0.001^{\dagger}$ \\
\hline \multicolumn{4}{|l|}{ Lymphovascular invasion } \\
\hline Positive ( $n=168$ ) & 163 & 5 & $<0.001^{\ddagger}$ \\
\hline Negative $(n=103)$ & 62 & 41 & \\
\hline
\end{tabular}

${ }^{\dagger}$ Two-sided $p$ value determined by $t$ test. ${ }^{*}$ Two-sided $p$ value determined by the $\chi^{2}$ test.

Expression of HER-3 in lung and breast cancer cell lines as determined by RT-PCR and immunoblotting. Expression of HER-3 mRNA was examined by RT-PCR in eight NSCLC and five breast cancer cell lines. HER-3 mRNA was detected in all cell lines (Figure 2(a)). Following sequence analysis, nucleotide sequence of cDNA fragments from the eight NSCLC cell lines matched that of HER-3: NM_001982 (http://blast.ncbi.nlm.nih.gov/Blast.cgi). No mutation was found.

HER-3 antibodies were raised in our lab following published protocols [4]-[6]. Specificity of the antibodies was determined by immunoblotting analysis. HER-3 was detected in all five breast cancer, but barely detected in one (H1437) of 8 lung cancer cell lines (Figure 2(b)). Interestingly, two protein bands (180- and 185-kDa) were recognized. The 185-kDa protein was highly expressed in breast cancer cell lines, MCF-1, MCF-7 and T47D. Using small hairpin RNA (shRNA), levels of both 180- and 185-kDa protein were markedly reduced in T47D cells, indicating that both proteins were HER-3 (Figure 2(c)). Moreover, both 180- and 185-kDa proteins were precipitated by HER-3 specific antibodies. The precipitates were analyzed by MALDI-TOF, and the peptide mass fingerprints of both protein bands matched HER-3: UniProtKB/Swiss-Prot: P21860, suggesting that the 185-kDa protein could be a post-translationally modified 180-kDa HER-3. Findings of HER-3 with various forms in normal tissues, e.g., stomach, large intestine and spleen, further support that HER-3 expression is physiologically controlled (Figure 2(d)).

The distinct discrepancy in levels of abundant mRNA and rarely detected protein in lung cancer cells suggested that HER-3 expression could also be pathophysiologically regulated. However, our previous study of eukaryotic elongation factor 2 (eEF2) showed that the total translation efficacy in LADC cells was in fact enhanced [25]. The present results implicated that inhibition of protein synthesis could be HER-3 gene specific. 


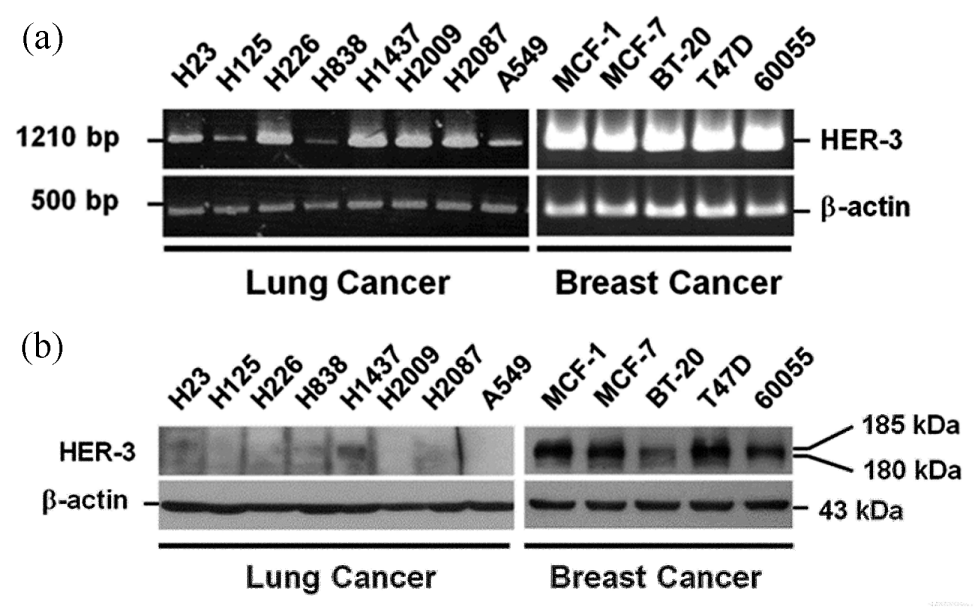

(c)

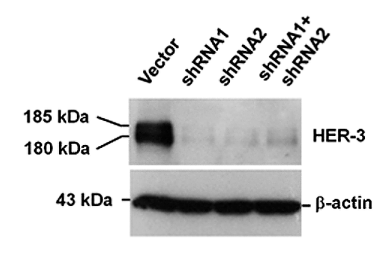

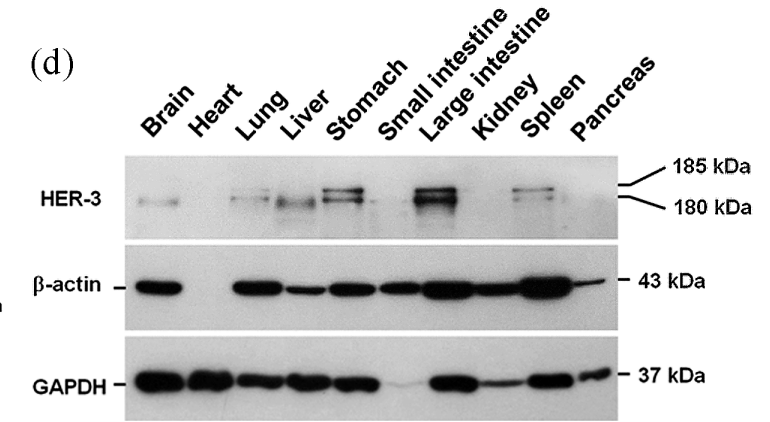

(d)

Figure 2. Expression of ErB3 in lung and breast cancer cells as detected by RT-PCR and immunoblotting. (a) Expression of HER-3 mRNA in 8 lung and 5 breast cancer cell lines as detected by RT-PCR. Expression of $\beta$-actin was used as a monitoring standard for the relative expression of HER-3 mRNA. (b) Expression of HER-3 protein in 8 lung and 5 breast cancer cell lines as detected by an immunoblotting analysis. $\beta$-actin served as a loading control. HER-3 was recognized in all 5 breast cancer cell lines. However, only a weak 180-kDa HER-3 was identified in H1437 of the 8 NSCLC cell lines. The 185-kDa protein was highly expressed in 3 breast cancer cell lines, MCF-1, MCF-7 and T47D. (c) The effect of small hairpin RNA (shRNA) on expression of HER-3 protein as determined by immunoblotting analysis. Addition of shRNA reduced both 180- and 185-kDa protein levels in T47D cells, indicating that HER-3 proteins could have two forms, and the $185-\mathrm{kDa}$ protein could be a post-translationally modified HER-3. (d) The immunoblotting analysis of HER-3 expression in normal murine tissues. HER-3 with various molecular weights was detected in the brain, lung, liver, stomach, large intestine and spleen, supporting the notion that expression of HER-3 is physiologically regulated.

We, therefore, used web programs to search for potential microRNA (miRNA), which might suppress HER-3 protein synthesis, and we identified miR-22 as one of the most probable candidates (Table 2). Unlike other miRNAs, which can be categorized into subfamilies, miR-22 is unique.

Addition of hsa-miR-22E to breast cancer cells reduces HER-3 protein levels, and ectopic expression of hsamiR-22 inhibitor in LADC cells increases HER-3 protein levels. As shown in Figure 3(a), addition of hsa-miR22 (ABM, Richmond, BC, Canada) or miR-22E reduced HER-3 protein expression in a time-dependent fashion in MCF-7 cells. Addition of miR-342 (a microRNA targeting at HER-2), however, did not affect HER-3 expression. Interestingly, miR-22E inhibited both 180- and 185-kDa HER-3 at the same rate, whereas miR-22 reduced the 180-kDa HER-3 first and then the 185-kDa protein at a slower rate, suggesting that miR-22E could simultaneously impede HER-3 protein biosynthesis and phosphorylation. Ectopic expression of miR-22 inhibitor (lentimiRa-Off-hsa-miR-22, ABM, Richmond, BC, Canada), on the other hand, increased both 180- and 185-kDa HER-3 protein bands in H23 and H1437 cells (Figure 3(b)), confirming that miR-22 could down-regulated HER-3 protein expression [17].

Using miR-22E mimics to inhibit HER-3 expression increased mobility, but reduced growth of T47D cells (Figures 4(a)-(c)). In contrast, using ectopic miR-22 inhibitor to augment HER-3 expression decreased movement of H23 cells (Figure 4(b) and Figure 4(c)). 
Table 2. Using online software programs, TargetScan6.0 (www.targetscan.org), miRDB (http://www.mirdb.org/), miRanda (www.microrna.org), miRBase (www.ebi.ac.uk), TarBase (http://diana.imis.athena-innovation.gr/), and RepTar (http://www.benoslab.pitt.edu/comir/), to search for the potential microRNA (miRNA), which could suppress HER-3 protein synthesis.

\begin{tabular}{|c|c|c|c|c|c|c|}
\hline Web softwares & TargetScan 6.0 & miRDB & miRanda & miRBase & TarBase & RepTar \\
\hline $\begin{array}{c}\text { Prospective } \\
\text { microRNAs I } \\
\text { (miR-17 family) }\end{array}$ & $\begin{array}{c}\text { hsa-miR-106a } \\
\text { hsa-miR-17 } \\
\text { has-miR-20b } \\
\text { hsa-miR-93 } \\
\text { hsa-miR-106b } \\
\text { hsa-miR-20a }\end{array}$ & $\begin{array}{l}\text { hsa-miR-106a } \\
\text { hsa-miR-17 } \\
\text { has-miR-20b } \\
\text { hsa-miR-93 }\end{array}$ & $\begin{array}{l}\text { hsa-miR-106a } \\
\text { hsa-miR-17 } \\
\text { has-miR-20b } \\
\text { hsa-miR-93 } \\
\text { hsa-miR-106b } \\
\text { hsa-miR-20a }\end{array}$ & $\begin{array}{l}\text { hsa-miR-106a } \\
\text { hsa-miR-17 } \\
\text { has-miR-20b } \\
\text { hsa-miR-93 } \\
\text { hsa-miR-106b } \\
\text { hsa-miR-20a }\end{array}$ & $\begin{array}{l}\text { hsa-miR-106a } \\
\text { hsa-miR-17 } \\
\text { has-miR-20b } \\
\text { hsa-miR-93 } \\
\text { hsa-miR-106b } \\
\text { hsa-miR-20a }\end{array}$ & $\begin{array}{c}\text { hsa-miR-106a } \\
\text { hsa-miR-17 } \\
\text { has-miR-20b } \\
\text { hsa-miR-93 } \\
\text { hsa-miR-106b } \\
\text { hsa-miR-20a }\end{array}$ \\
\hline $\begin{array}{l}\text { Prospective microRNAs } \\
\text { II (miR-130 family) }\end{array}$ & $\begin{array}{l}\text { hsa-miR-130a } \\
\text { hsa-miR-130b }\end{array}$ & hsa-miR-130b & $\begin{array}{l}\text { hsa-miR-130a } \\
\text { hsa-miR-130b }\end{array}$ & & $\begin{array}{l}\text { hsa-miR-130a } \\
\text { hsa-miR-130b }\end{array}$ & hsa-miR-130a \\
\hline $\begin{array}{c}\text { Prospective } \\
\text { microRNAs III } \\
\text { (miR-148 family) }\end{array}$ & $\begin{array}{c}\text { hsa-miR-148a } \\
\text { hsa-miR-148b } \\
\text { hsa-miR-152 }\end{array}$ & $\begin{array}{l}\text { hsa-miR-148a } \\
\text { hsa-miR-148b } \\
\text { hsa-miR-152 }\end{array}$ & $\begin{array}{l}\text { hsa-miR-148a } \\
\text { hsa-miR-148b } \\
\text { hsa-miR-152 }\end{array}$ & & $\begin{array}{l}\text { hsa-miR-148a } \\
\text { hsa-miR-148b } \\
\text { hsa-miR-152 }\end{array}$ & \\
\hline $\begin{array}{l}\text { Prospective microRNAs } \\
\text { IV (miR-205 family) }\end{array}$ & hsa-miR-205 & & hsa-miR-205 & hsa-miR-205 & hsa-miR-205 & \\
\hline $\begin{array}{l}\text { Prospective microRNAs } \\
\text { V (miR-22 family) }\end{array}$ & hsa-miR-22 & & hsa-miR-22 & hsa-miR-22 & hsa-miR-22 & \\
\hline $\begin{array}{l}\text { Prospective microRNAs } \\
\text { VI (miR-515 family) }\end{array}$ & hsa-miR-519d & $\begin{array}{l}\text { hsa-miR-519d } \\
\text { hsa-miR-520b } \\
\text { hsa-miR-520d-3p }\end{array}$ & $\begin{array}{l}\text { hsa-miR-519d } \\
\text { hsa-miR-520b } \\
\text { hsa-miR-520d-3p }\end{array}$ & & $\begin{array}{c}\text { hsa-miR-519d } \\
\text { hsa-miR-520b } \\
\text { hsa-miR-520d-3p }\end{array}$ & $\begin{array}{c}\text { hsa-miR-520b } \\
\text { hsa-miR-520d-3p }\end{array}$ \\
\hline
\end{tabular}

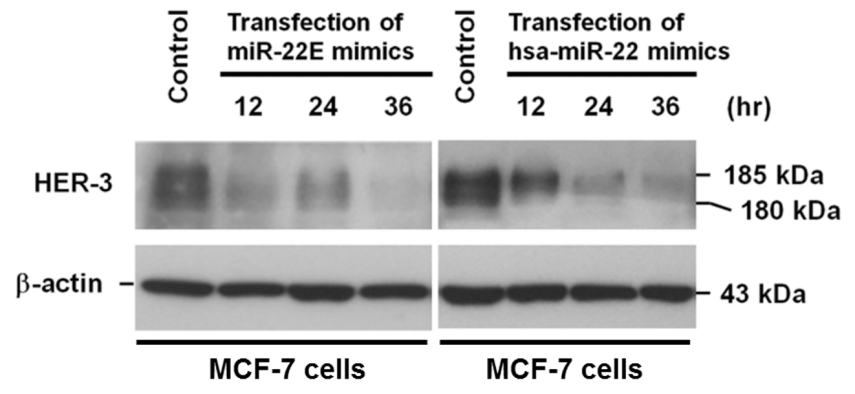

(a)

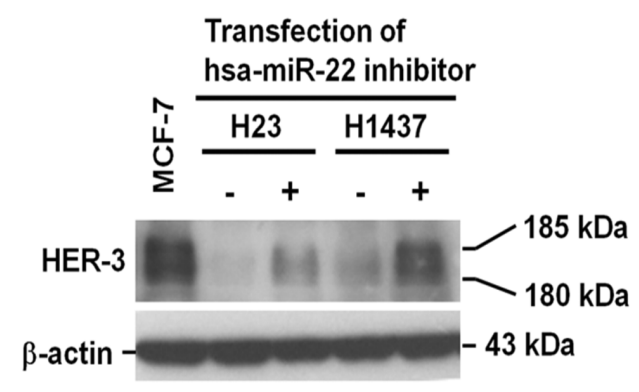

(b)

Figure 3. The effect of miR-22E mimics, hsa-miR-22 mimics and hsa-miR-22 inhibitor on HER-3 expression. (a) The effect of synthetic miR-22E and hsa-miR-22 on HER-3 protein expression in MCF-7 cancer cells as determined by immunoblotting analysis. Ectopic expression of miR-22E mimics inhibited both 180- and 185-kDa HER-3 at about the same rate, whereas miR-22 reduced the 180-kDa HER-3 first and then the 185-kDa protein at a slower rate. Addition of miR-342 (a miRNA targeting at HER-2), used as a negative control, did not affect expression of HER-3 [39] [40]. (b) Addition of ectopic miR-22 inhibitor (lentimiRa-Off-hsa-miR-22) increased both 180- and 185-kDa HER-3 proteins in H23 and H1437 cells as determined by immunoblotting analysis, confirming our previous results that miR-22 reduced HER-3 protein expressions, and the miR-22 inhibitor reversed the suppressive effect by inhibiting the function of miR-22.

Addition of EGF increases expression of miR-22H and miR-22L in cancer cells. In vitro, addition of EGF or concurrent addition of EGF and HGF significantly increased EGFR, but reduced HER-3 expression in BT-20 cells (Figure 5(a)). Addition of HGF alone, however, only increased molecular shifting of EGFR, but not HER-3 expression. Interestingly, by showing that challenge cells with EGF or with EGF and HGF could simultaneously increase expression of miR-22H and miR-22L (Figure 5(b)), which in turn reduced HER-3 protein levels, our data confirmed that miR-22 could inhibit HER-3 translation. Cell biology studies demonstrated that EGF induced cell growth (Figure 5(c), upper panel), whereas HGF activated cell mobility (Figure 5(c), lower panel). Moreover, tissue levels of heparin-binding EGF-like growth factor (HB-EGF) in patients with positive miR-22 expressions (163 $\pm 21.85 \mathrm{pg} / \mathrm{mg} ; \mathrm{n}=53$ ) were significantly higher than those with negative miR-22 expressions (76 $\pm 6.7 \mathrm{pg} / \mathrm{mg} ; \mathrm{n}=10$ ) (Figure 5(d)), also supported our in vitro observation. 
(a)
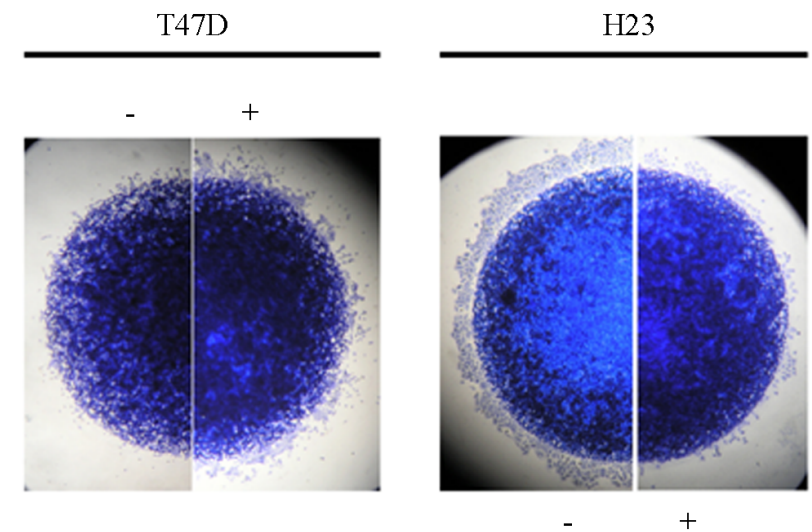

Cell line

miR-22E-mimics

(b)

miR-22E
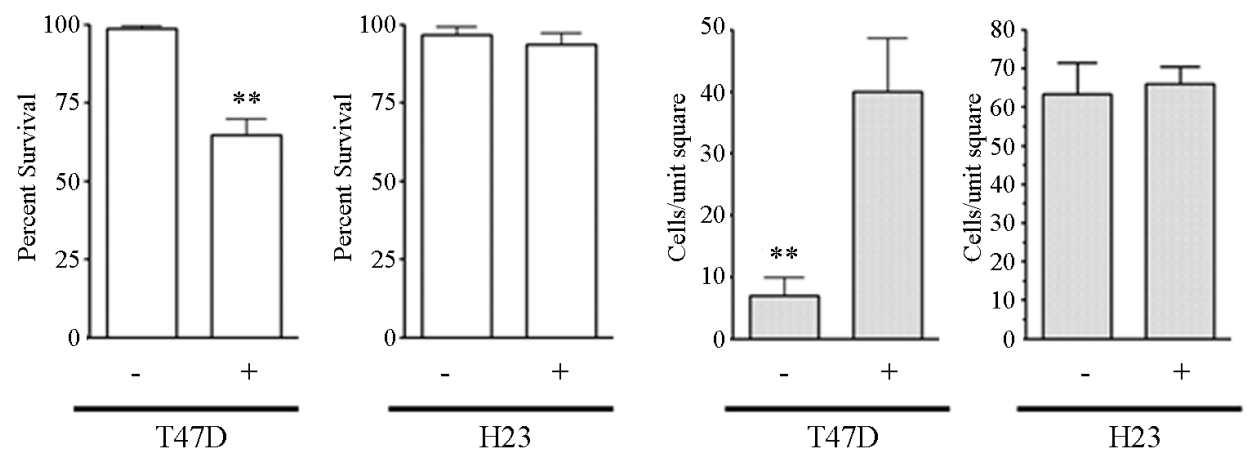

(c)

miR-22

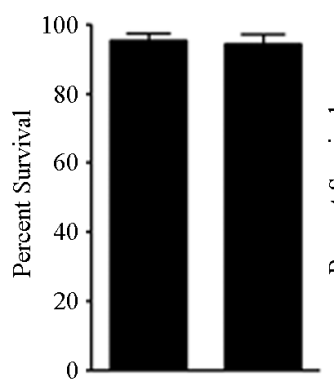

Inhibitor

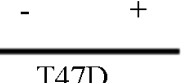

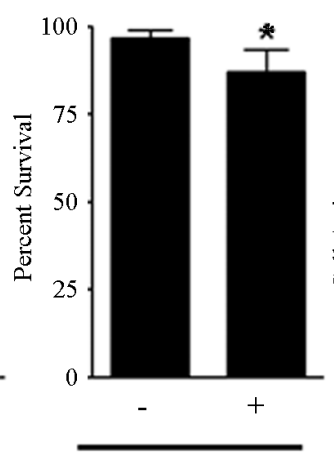

$\mathrm{H} 23$

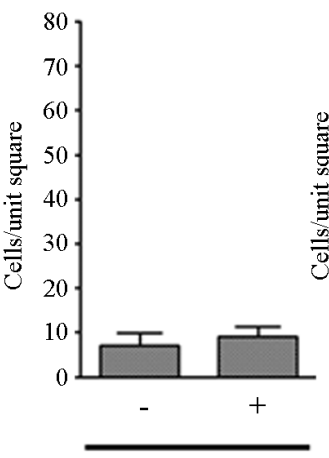

T47D

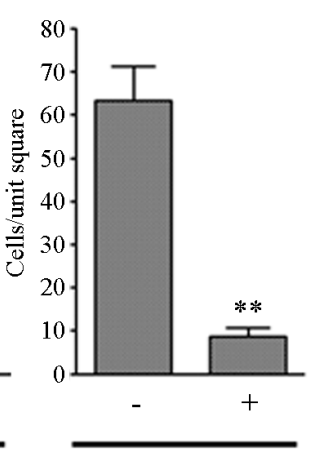

$\mathrm{H} 23$

Figure 4. The effect of miR-22 and miR-22 inhibitor on cell proliferation and movement. (a) The respective effect of miR-22 and miR-22 inhibitor on cell movement out of agarose gel droplet. T47D cells, which highly expressed HER-3, did not readily move out of the agarose (the left panel). In the presence of miR-22E, the cells were more readily to move out of the agarose (the right panel). On the other hand, H23 cells, which hardly expressed HER-3, readily moved out of the agarose (the left panel). In lentimiRa-Off-hsa-miR-22 transfected H23 cells, expression of ectopic miR-22 inhibitor reduced cells moving out of the agarose (the right panel). (b) The statistical summaries of the effect of miR-22 on the cell proliferation and movement. (c) The statistical summaries of the effect of miR-22 inhibitor on the cell proliferation and movement.

\section{Discussion}

Our results show that although HER-3 mRNA is abundantly detected in LADC cells, HER-3 protein is rarely identified. Compared to breast cancer cells, which highly expressed HER-3 proteins, miR-22H which contained an extra 13 bases, 5'-TGTGTTCAGTGGT-3' (miR-22E), at 3'-end was found to inhibit HER-3 protein synthesis in LADC cells. However, the source of miR-22E sequences was not identified in the near vicinity of miR-22 gene by an online software BLAST (http://blast.ncbi.nlm.nih.gov/Blast.cgi), suggesting that miR-22H could be a 
(a)

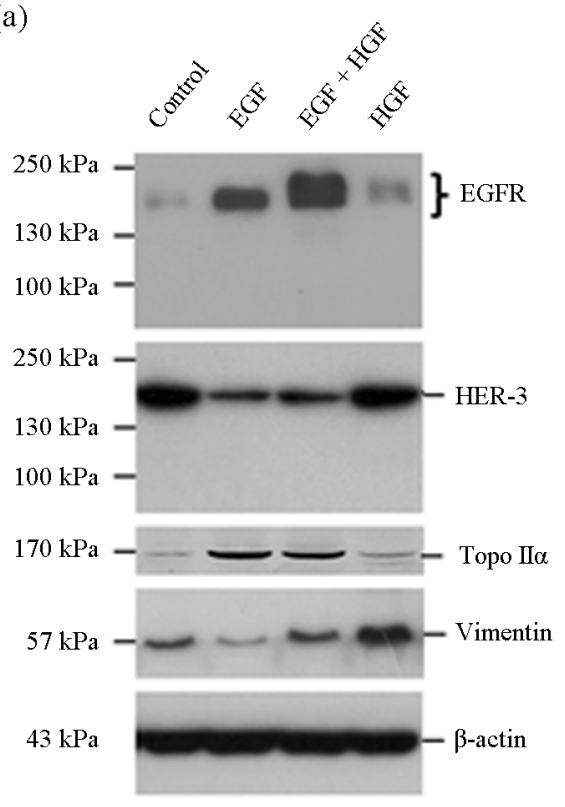

(b)

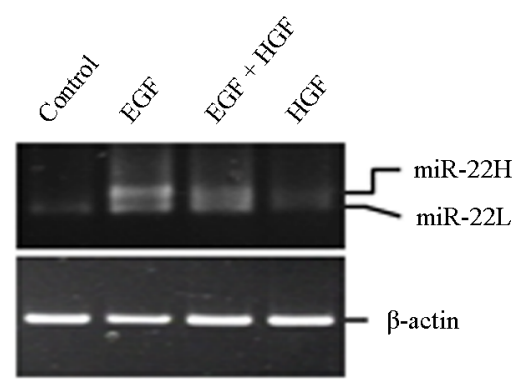

(c)

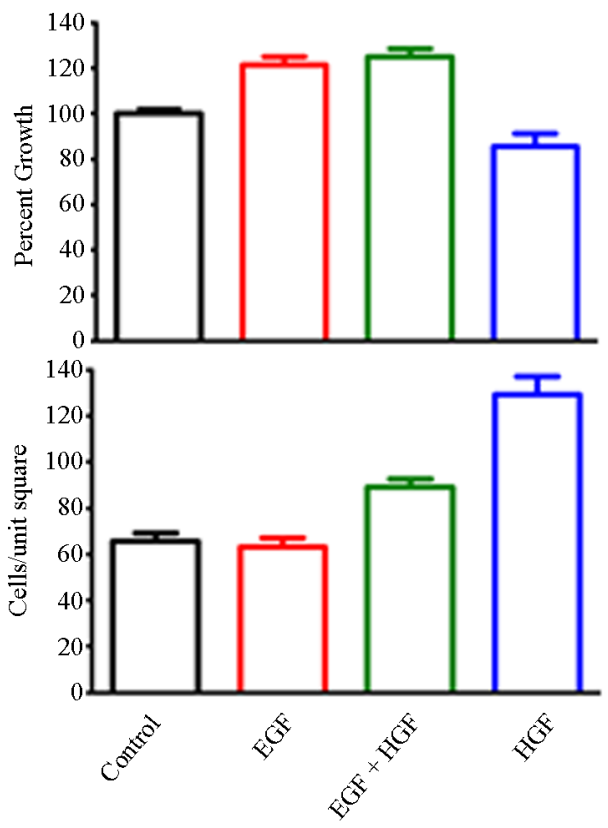

(d)

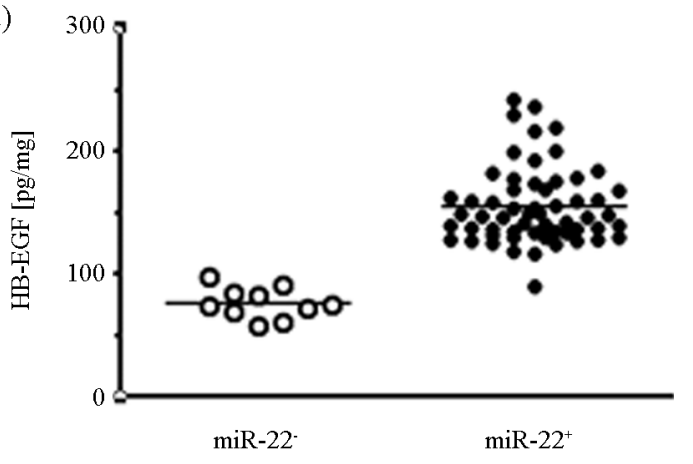

Figure 5. The effect of EGF and HGF on the expression of EGFR, HER-3 and miR-22 as well as cell behavior of BT-20 cells. (a) The effect of EGF, HGF or the combination of both growth factors on the expression of EGFR, HER-3, proliferation marker topoisomerase II-alpha (Topo II $\alpha$ ), and EMT marker vimentin in BT-20 cells as determined by the immunoblotting analysis. In vitro, treatment with EGF alone or concurrent treatment with EGF and HGF significantly increased EGFR and topo II $\alpha$, but reduced HER-3 expression. Addition of EGF alone, however, reduced vimentin expression. Addition of HGF alone, on the other hand, increased not only the molecular weight shifting of EGFR, but also the vimentin expression. (b) The effect of EGF, HGF or the combination of both growth factors on the expression of miR-22. When cells were challenged with EGF or simultaneously with EGF and HGF, expression of both miR-22H and miR-22L were elevated, which correlated with the increased EGFR expression, but with reduced HER-3 protein levels, validating our data that miR-22 repressed HER-3 protein expression. (c) The effect of EGF, HGF or the combination of both growth factors on the cell behaviors. Addition of EGF induced cell growth, whereas addition of HGF activated cell mobility. (d) Tissue levels of heparin-binding EGF-like growth factor (HB-EGF) in patients with miR-22 expressions (163 \pm 21.85 pg/mg; $\mathrm{n}=53)$ were significantly higher than those with no miR-22 expression $(76 \pm 6.7 \mathrm{pg} / \mathrm{mg} ; \mathrm{n}=10)$.

product of post-transcriptional modifications, e.g., maturation [26] and splicing [27]. Pathologically, miR-22E was highly detected in surgical specimens of LADC patients, and the miR-22E expression level was clearly correlated with higher incidence of early tumor recurrence and increased metastasis, which ultimately reflected in worse clinical outcomes (Table 2).

In vitro, miR-22E had a higher efficiency in reducing HER-3 protein levels than miR-22. Furthermore, miR22E inhibited both 180- and 185-kDa HER-3 at the same rate, while miR-22 reduced 180-kDa HER-3 first and then the $185-\mathrm{kDa}$ protein, indicating that miR-22E was functional and could simultaneously interrupt HER-3 
protein biosynthesis and phosphorylation. Inhibition of HER-3 protein synthesis reduced proliferation, but increased mobility of T47D cells. Ectopic expression of hsa-miR-22 inhibitor, on the other hand, increased levels of both 180- and 185-kDa HER-3, verifying our results that miR-22 could concurrently interfere HER-3 protein synthesis and phosphorylation. Although increase of HER-3 expression reduced cell movement, it did not affect cell growth, implicating that up-stream factors were involved in balancing the expression of miR-22 and HER-3, and the cell behaviors.

In fact, increased miR-22 levels have been shown to enhance metastatic potential of MCF-7 breast cancer cells by modulating expression of methylcytosine dioxygenases (also known as ten eleven translocases, TETs), the enzymes which are essential for DNA demethylation [28]. Without proper DNA demethylation, miR-200 synthesis was inhibited to favor expression of metastasis-associated genes [26], e.g., platelet-derived growth factor (PDGF) [29], interleukin-8 (IL-8), chemokine (C-X-C motif) ligand 1 (CXCL-1) [30], and vimentin [31], but to reduce expression of metastasis-suppressive genes, e.g., E-cadherin, SEC23A (Sec23 homolog A of S. cerevisiae), insulin-like growth factor binding protein 4 (igfbp4) and tubulointerstitial nephritis antigen-like 1 (tinagl1) [32]. Imbalance of metastasis-associated over metastasis-suppressive gene expressions would aggravate epithelial-to-mesenchymal transition (EMT) and distant metastasis of tumor cells. However, miR-22 expression was not emphasized in those studies.

Bar and Dikstein showed that through activation of phosphoinositide 3-kinase (PI3K) and production of phosphatidylinositol-3,4,5-triphosphate (PIP3), AKT could up-regulate miR-22 expression to facilitate cell migration [19]. The receptor tyrosine kinase, which mediated PI3K activation, however, was not specified. The present study sheds some light on this issue, and suggests that activated EGFR is accountable for the increase of miR-22 expression [33]. It was further shown that by interacting with argonaute 2 (AGO2), EGFR which was activated by hypoxia could inhibit Dicer activity and miRNA maturation [34]. Two forms of EGFR activation have been demonstrated [4] [34]: one form is through EGF to increase EGFR synthesis and tyrosine phosphorylation; the other form is to increase phosphorylation of serine/threonine residues via HGF [3] [4]. Through c-MET, HGF activates FAK, PI3K and AKT, as well.

Interestingly, hypoxia also increases expression of heparin-binding EGF-like growth factor (HB-EGF) [35] and gefitinib resistance in NSCLC cells [36]. All these data considered together supported our current results and several previous studies, which suggested that EGF, TGF- $\alpha$, IGF, and FGF respectively regulated miR-22 expression [18] [19]. Moreover, our results showed that exogenously added EGF induced expression of EGFR, and miR-22, which, on the other hand, reduced protein levels of HER-3, a prospective decoy receptor that counteracted the effect of EGFR, indicating that there could be a possible feedback circuit to orchestrate intracellular events to benefit cancer cell survival and to overcome the harsh microenvironments [6] [13] [37].

It is worth noting that although our data show that HER-3 mRNA is highly expressed in LADC cells [10], the HER-3 protein is rarely identified. On the contrary, miR-22 is frequently detected in LADC specimens, especially those from patients who have smoking history. Statistical analyses showed that the increased miR-22 expression was closely associated with the malignant disease progression, including lymphovascular invasion and the advanced stages of disease. In vitro, miR-22 inhibits HER-3 protein expression, and increases cell's traversing ability, ratifying our pathologic findings [3] [4]. In fact, when evaluating microRNA expression profiles, Park et al. had elegantly classified 60 multifarious cancer cell lines into epithelial and mesenchymal subtypes, and found that the miR-200 family was specific for epithelial phenotype. They also showed that the miR-155, miR-210 and miR-22 were unique for the mesenchymal phenotype [31], which further supported our data that miR-22 expression favored EMT, and cancer cell metastasis. However, other explanations are possible. For instance, we have not determined the role of miR-155 or miR-210 in the LADC, which are up-regulated in many different cancer types [31] [38]. In an ongoing study, we are checking these aspects. It should be noted that rapid cancer cell growth during disease progression often resulted in reduced supply of oxygen as well as nutrients, and the subsequent accumulation of tumor cell-specific metabolites and growth factors in tumor nests. Our findings may provide some explanations for how changes in microenvironments affect gene expression patterns of tumor cells, including microRNA as well as intracellular detoxification-, mitochondrial transport-, DNA repair-, and membrane receptor-related proteins, to benefit survival and potential metastasis of cancer cells [5]-[9], which in turn reflect in poor prognosis. Our data clearly show that parameters for determining tumor cell behaviors and the prospective therapeutic efficacies in cancer patients are multifactorial, involving several levels of gene expression regulations.

In conclusion, our results suggest that HER-3 may act as a decoy receptor of EGFR family in LADC cells, 
and miR-22, especially miR22-E, inhibits HER-3 protein expression to promote disease progression. Expression of miR-22 could be a counter-feedback to maintain EGFR function.

\section{Acknowledgements}

The authors thank Miss Chien-Yu Chang for technical assistance. This study was supported by Cancer Excellent Research System (DOH102-TD-111-005, Taiwan), the National Science Council (103-2320-B-005-009, Taipei, Taiwan) and the Comprehensive Academic Promotion Projects (NCHU 1025025, Ministry of Education, Taiwan) and the Ministry of Education, Taiwan, under the ATU plan.

\section{Author Contributions}

H.Y.F., C.Y.C. collected patient's data, performed data analysis and arranged results for the manuscript; S.H.C., L.S.W., T.Y.L. operated experiments and data analysis; K.C.C. perceived the idea, designed and guided the experiment procedures, as well as prepared and wrote the manuscript.

\section{Conflicts of Interest Statement}

The authors declare no potential conflicts of interest.

\section{References}

[1] Huang, S.-F., Liu, H.-P., Li, L.-H., Ku, Y.-C., Fu, Y.-N., Tsai, H.-Y., et al. (2004) High Frequency of Epidermal Growth Factor Receptor Mutations with Complex Patterns in Non-Small Cell Lung Cancers Related to Gefitinib Responsiveness in Taiwan. Clinical Cancer Research, 10, 8195-8203. http://dx.doi.org/10.1158/1078-0432.CCR-04-1245

[2] Wu, J.-Y., Wu, S.-G., Yang, C.-H., Chang, Y.-L., Chang, Y.-C., Hsu, Y.-C., et al. (2011) Comparison of Gefitinib and Erlotinib in Advanced NSCLC and the Effect of EGFR Mutations. Lung Cancer, 72, 205-212. http://dx.doi.org/10.1016/j.lungcan.2010.08.013

[3] Chen, J.-T., Lin, T.-S., Chow, K.-C., Huang, H.-H., Chiou, S.-H., Chiang, S.-F., et al. (2006) Cigarette Smoking Induces Overexpression of Hepatocyte Growth Factor in Type II Pneumocytes and Lung Cancer cells. American Journal of Respiratory Cell and Molecular Biology, 34, 264-273. http://dx.doi.org/10.1165/rcmb.2005-0117OC

[4] Chen, J.-T., Huang, C.-Y., Chiang, Y.-Y., Chen, W.-H., Chiou, S.-H., Chen, C.-Y., et al. (2008) HGF Increases Cisplatin Resistance via Down-Regulation of AIF in Lung Cancer Cells. American Journal of Respiratory Cell and Molecular Biology, 38,559-565. http://dx.doi.org/10.1165/rcmb.2007-00010C

[5] Hsu, N.Y., Ho, H.C., Chow, K.C., Lin, T.-Y., Shih, C.-S., Wang, L.-S., et al. (2001) Overexpression of Dihydrodiol Dehydrogenase as a Prognostic Marker of Non-Small Cell Lung Cancer. Cancer Research, 61, 2727-2731.

[6] Chiang, Y.-Y., Chen, S.-L., Hsiao, Y.-T., Huang, C.-H., Lin, T.-Y., Chiang, I.-P., et al. (2009) Nuclear Expression of Dynamin-Related Protein 1 in Lung Adenocarcinomas. Modern Pathology, 22, 1139-1150. http://dx.doi.org/10.1038/modpathol.2009.83

[7] Fang, H.Y., Chang, C.L., Hsu, S.H., Huang, C.Y., Chiang, S.F., Chiou, S.H., et al. (2010) ATPase Family AAA Domain-Containing 3A Is a Novel Anti-Apoptotic Factor in Lung Adenocarcinoma Cells. Journal of Cell Science, 123, 1171-1180.

[8] You, W.-C., Chiou, S.-H., Huang, C.-Y., Chiang, S.-F., Yang, C.-L., Sudhakar, J.N., et al. (2013) Mitochondrial Protein ATPase Family, AAA Domain Containing 3A Correlates with Radioresistance in Glioblastoma. Neuro-Oncology, 15, 1342-1352. http://dx.doi.org/10.1093/neuonc/not077

[9] Chen, D.-R., Chu, C.-Y., Chen, C.-Y., Yang, H.-C., Chiang, Y.-Y., Lin, T.-Y., et al. (2008) Expression of Short Form Oncostatin M Receptor as a Decoy Receptor in Lung Adenocarcinomas. The Journal of Pathology, 215, $290-299$. http://dx.doi.org/10.1002/path.2361

[10] Chen, H.-Y., Yu, S.-L., Chen, C.-H., Chang, G.-C., Chen, C.-Y., Yuan, A., et al. (2007) A Five-Gene Signature and Clinical Outcome in Non-Small-Cell Lung Cancer. The New England Journal of Medicine, 356, 11-20. http://dx.doi.org/10.1056/NEJMoa060096

[11] Olayioye, M.A., Neve, R.M., Lane, H.A. and Hynes, N.E. (2000) The ErbB Signaling Network: Receptor Heterodimerization in Development and Cancer. The EMBO Journal, 19, 3159-3167. http://dx.doi.org/10.1093/emboj/19.13.3159

[12] Sierke, S.L., Cheng, K., Kim, H.H. and Koland, J.G. (1997) Biochemical Characterization of the Protein Tyrosine kinase Homology Domain of the ErbB3 (HER3) Receptor Protein. Biochemical Journal, 322, 757-763. 
[13] Steinberg, F., Zhuang, L., Beyeler, M., Kälin, R.E., Mullis, P.E., Brändli, A.W., et al. (2010) The FGFRL1 Receptor Is Shed from Cell Membranes, Binds Fibroblast Growth Factors (FGFs), and Antagonizes FGF Signaling in Xenopus Embryos. The Journal of Biological Chemistry, 285, 2193-2202. http://dx.doi.org/10.1074/jbc.M109.058248

[14] Bogdan, S. and Klämbt, C. (2001) Epidermal Growth Factor Receptor Signaling. Current Biology, 11, R292-R295. http://dx.doi.org/10.1016/S0960-9822(01)00167-1

[15] Hellyer, N.J., Cheng, K. and Koland, J.G. (1998) ErbB3 (HER3) Interaction with the p85 Regulatory Subunit of Phosphoinositide 3-Kinase. Biochemical Journal, 333, 757-763.

[16] Bartel, D.P. (2009) MicroRNAs: Target Recognition and Regulatory Functions. Cell, 136, 215-233. http://dx.doi.org/10.1016/j.cell.2009.01.002

[17] Patel, J.B., Appaiah, H.N., Burnett, R.M., Bhat-Nakshatri, P., Wang, G., Mehta, R., et al. (2011) Control of EVI-1 Oncogene Expression in Metastatic Breast Cancer Cells through microRNA miR-22. Oncogene, 30, 1290-1301. http://dx.doi.org/10.1038/onc.2010.510

[18] Larue, L. and Bellacosa, A. (2005) Epithelial-Mesenchymal Transition in Development and Cancer: Role of Phosphatidylinositol 3’Kinase/AKT Pathways. Oncogene, 24, 7443-7454. http://dx.doi.org/10.1038/sj.onc.1209091

[19] Bar, N. and Dikstein, R. (2010) miR-22 Forms a Regulatory Loop in PTEN/AKT Pathway and Modulates Signaling Kinetics. PLoS One, 5, e10859. http://dx.doi.org/10.1371/journal.pone.0010859

[20] Kasahara, K., Arao, T., Sakai, K., Matsumoto, K., Sakai, A., Kimura, H., et al. (2010) Impact of Serum Hepatocyte Growth Factor on Treatment Response to Epidermal Growth Factor Receptor Tyrosine Kinase Inhibitors in Patients with Non-Small Cell Lung Adenocarcinoma. Clin Cancer Res, 16, 4616-4624. http://dx.doi.org/10.1158/1078-0432.CCR-10-0383

[21] Yano, S., Wang, W., Li, Q., Matsumoto, K., Sakurama, H., Nakamura, T., et al. (2008) Hepatocyte Growth Factor Induces Gefitinib Resistance of Lung Adenocarcinoma with Epidermal Growth Factor Receptor-Activating Mutations. Cancer Research, 68, 9479-9487. http://dx.doi.org/10.1158/0008-5472.CAN-08-1643

[22] Mountain, C.F. (1997) Revisions in the International System for Staging Lung Cancer. Chest, 111, 1710-1717. http://dx.doi.org/10.1378/chest.111.6.1710

[23] Kaplan, E.L. and Meier, P. (1958) Nonparametric Estimation from Incomplete Observations. Journal of the American Statistical Association, 53, 457-481. http://dx.doi.org/10.1080/01621459.1958.10501452

[24] Mantel, N. (1966) Evaluation of Survival Data and Two New Rank Order Statistics Arising in Its Consideration. Cancer Chemotherapy Reports, 50, 163-170.

[25] Chen, C.-Y., Fang, H.-Y., Chiou, S.-H., Yi, S.-E., Huang, C.-Y., Chiang, S.-F., et al. (2011) Sumoylation of Eukaryotic Elongation Factor 2 Is Vital for Protein Stability and Anti-Apoptotic Activity in Lung Adenocarcinoma Cells. Cancer Science, 102, 1582-1589. http://dx.doi.org/10.1111/j.1349-7006.2011.01975.X

[26] Lee, Y., Jeon, K., Lee, J.T., Kim, S. and Kim, V.N. (2002) MicroRNA Maturation: Stepwise Processing and Subcellular Localization. The EMBO Journal, 21, 4663-4670. http://dx.doi.org/10.1093/emboj/cdf476

[27] Flynt, A.S., Greimann, J.C., Chung, W.J., Lima, C.D. and Lai, E.C. (2010) MicroRNA Biogenesis via Splicing and Exosome-Mediated Trimming in Drosophila. Molecular Cell, 38, 900-907. http://dx.doi.org/10.1016/j.molcel.2010.06.014

[28] Song, S.J., Poliseno, L., Song, M.S., Ala, U., Webster, K., Ng, C., et al. (2013) MicroRNA-Antagonism Regulates Breast Cancer Stemness and Metastasis via TET-Family-Dependent Chromatin Remodeling. Cell, 154, 311-324. http://dx.doi.org/10.1016/j.cell.2013.06.026

[29] Kong, D.J., Li, Y.W., Wang, Z.W., Banerjee, S., Ahmad, A., Choi Kim, H.-R. and Sarkar, F.H. (2009) miR-200 Regulates PDGF-D-Mediated Epithelial-Mesenchymal Transition, Adhesion, and Invasion of Prostate Cancer Cells. Stem Cells, 27, 1712-1721. http://dx.doi.org/10.1002/stem.101

[30] Pecot, C.V., Rupaimoole, R., Yang, D., Akbani, R., Ivan, C., Lu, C.H., et al. (2013) Tumour Angiogenesis Regulation by the miR-200 Family. Nature Communications, 4, Article Number: 2427. http://dx.doi.org/10.1038/ncomms3427

[31] Park, S.M., Gaur, A.B., Lengyel, E. and Peter, M.E. (2008) The miR-200 Family Determines the Epithelial Phenotype of Cancer Cells by Targeting the E-Cadherin Repressors ZEB 1 and ZEB 2 . Genes \& Development, 22, 894-907. http://dx.doi.org/10.1101/gad.1640608

[32] Korpal, M., Ell, B.J., Buffa, F.M., Ibrahim, T., Blanco, M.A., Celià-Terrassa, T., et al. (2011) Direct Targeting of Sec23a by miR-200s Influences Cancer Cell Secretome and Promotes Metastatic Colonization. Nature Medicine, 17, 1101-1108. http://dx.doi.org/10.1038/nm.2401

[33] Hennessy, B.T., Smith, D.L., Ram, P.T., Lu, Y. and Mills, G.B. (2005) Exploiting the PI3K/AKT Pathway for Cancer Drug Discovery. Nature Reviews Drug Discovery, 4, 988-1004. http://dx.doi.org/10.1038/nrd1902 
[34] Shen, J., Xia, W.Y., Khotskaya, Y.B., Huo, L.F., Nakanishi, K., Lim, S.-O., et al. (2013) EGFR Modulates microRNA Maturation in Response to Hypoxia through Phosphorylation of AGO2. Nature, 497, 383-387. http://dx.doi.org/10.1038/nature12080

[35] Jin, K., Mao, X.O., Sun, Y., Xie, L., Jin, L., Nishi, E., et al. (2002) Heparin-Binding Epidermal Growth Factor-Like Growth Factor: Hypoxia-Inducible Expression in Vitro and Stimulation of Neurogenesis in Vitro and in Vivo. The Journal of Neuroscience, 22, 5365-5373.

[36] Minakata, K., Takahashi, F., Nara, T., Hashimoto, M., Tajima, K., Murakami, A., et al. (2012) Hypoxia Induces Gefitinib Resistance in Non-Small-Cell Lung Cancer with Both Mutant and Wild-Type Epidermal Growth Factor Receptors. Cancer Science, 103, 1946-1954. http://dx.doi.org/10.1111/j.1349-7006.2012.02408.x

[37] Tavaluc, R.T., Hart, L.S., Dicker, D.T. and El-Deiry, W.S. (2007) Effects of Low Confluency, Serum Starvation and Hypoxia on the Side Population of Cancer Cell Lines. Cell Cycle, 6, 2554-2562. http://dx.doi.org/10.4161/cc.6.20.4911

[38] Yanaihara, N., Caplen, N., Bowman, E., Seike, M., Kumamoto, K., Yi, M., et al. (2006) Unique MicroRNA Molecular Profiles in Lung Cancer Diagnosis and Prognosis. Cancer Cell, 9, 189-198. http://dx.doi.org/10.1016/j.ccr.2006.01.025

[39] Chiang, Y.Y., Chow, K.C., Lin, T.Y., Chiang, I.P. and Fang, H.Y. (2014) Hepatocyte Growth Factor and HER2/Neu Downregulate Expression of Apoptosis-Inducing Factor in Non-Small Cell Lung Cancer. Oncology Reports, 31, 597604.

[40] Lowery, A.J., Miller, N., Devaney, A., McNeill, R.E., Davoren, P.A., Lemetre, C., et al. (2009) MicroRNA Signatures Predict Oestrogen Receptor, Progesterone Receptor and HER2/Neureceptor Status in Breast Cancer. Breast Cancer Research, 11, R27. http://dx.doi.org/10.1186/bcr2257 